\title{
MANAGEMENT OF AXILLARY DEFECT OF HIDRADENITIS SUPPURATIVA USING KEYSTONE DESIGN ISLANDED PERFORATOR FLAP- A SIMPLE AND DURABLE OPTION
}

\author{
Aravind Lakshmana Rao', Oliver Clement Lobo ${ }^{2}$
}

${ }_{1}^{1}$ Associate Professor, Department of Plastic Surgery, Father Muller Medical College, Mangalore.

2Postgraduate Resident, Department of Dermatology, Father Muller Medical College, Mangalore. \begin{abstract}
\section{BACKGROUND}

Hidradenitis suppurativa is a chronic cutaneous disease involving recurrent abscesses, fistulating sinus tracts and scarring. The most common sites involved include the axilla, inguinoperineal region and breasts. The disease causes considerable discomfort to the patient due to recurrent abscess and sinus tract with discharge. Medical management will only give temporary relief in resistant disease and surgical excision followed by suitable cover promises a better outcome and hence better quality of life to the patients with hidradenitis suppurativa. We describe a local fasciocutaneous flap- the keystone design, islanded, perforator-based flap for the reconstruction of axillary defects following wide excision of hidradenitis of axilla. This method provides a durable cover for the axillary region.

The aim of this article was to study the effectiveness of keystone flap in reconstruction of axillary defects after excision of hidradenitis suppurativa of the axilla.
\end{abstract}

\section{MATERIALS AND METHODS}

This case series involves study of 8 patients undergoing keystone flap for axillary defect following wide excision of skin with hidradenitis suppurativa from 2013 to 2015. Patient's demographic data, medical histories, comorbidities, defect characteristics, hospitalisation, complications and follow-up were evaluated and presented as uncontrolled case series.

\section{RESULTS}

Ages of the subjects were ranging from 18 to 55 years with an average of 34.13 years. Six patients had bilateral involvement and remaining two patients had unilateral involvement of the axilla. Fourteen keystone flaps were done for these patients including unilateral and bilateral disease. Surgery was done in two stages for patients with bilateral involvement with a gap of 2 months. The average intraoperative time was 58.5 mins (range 35 - 75 mins). Suction drain was kept in all the cases for a period of 5 days in all patients. A padded dressing with aeroplane slab was applied in all the patients for 5 days to facilitate wound immobilisation and rest. Wound was inspected on 3rd postoperative day. All the keystone flaps survived giving a durable cover to the axilla. However, two patients had wound infection, which subsided with dressings and appropriate antibiotics. The average duration of hospital stay of patients was 6.5 days. Sutures were removed after 2 weeks and patients were followed up for 6 months every month. All the flaps survived facilitating full range of shoulder movement. The patients showed good symptomatic relief from pain and pus discharge. Recurrence was not noted in any of these patients within the period of 6 months.

\section{CONCLUSION}

Keystone flap is a relatively simple local flap, which provides suitable cover to the axilla after excision of hidradenitis suppurativa and facilitates normal range of shoulder movement after surgery.

\section{KEYWORDS}

Keystone Flap; Hidradenitis Suppurativa; Perforator Flap; Reconstruction of Axilla.

HOW TO CITE THIS ARTICLE: Rao AL, Lobo OC. Management of axillary defect of hidradenitis suppurativa using keystone design islanded perforator flap- a simple and durable option. J. Evolution Med. Dent. Sci. 2018;7(02):218-221, DOI: $10.14260 /$ jemds/2018/49

\section{BACKGROUND}

Hidradenitis suppurativa is a chronic and recurrent inflammation of the skin and subcutaneous tissue. It originates from the apocrine glands and is characterised by suppuration and mucopurulent secretion, fistulae, unaesthetic scars and cicatricial retractions. ${ }^{1}$ The disease was first described in 1864 by Verneuil, a surgeon who identified

'Financial or Other Competing Interest': None.

Submission 23-11-2017, Peer Review 19-12-2017,

Acceptance 26-12-2017, Published 08-01-2018.

Corresponding Author:

Dr. Oliver Clement Lobo,

\#217, Don Bosco Boys Hostel,

Father Muller Medical College,

Kankanady, Mangalore-575002.

E-mail: oliverlobo2@gmail.com

DOI: $10.14260 /$ jemds/2018/49

involvement of sweat glands in the pathophysiology of the disease. ${ }^{1}$ The causative factors include diabetes, smoking and immunosuppression. ${ }^{2}$

There are painful, deep seated inflamed lesions in the apocrine gland-bearing areas of the body; most commonly the axillary, inguinal and anogenital regions. ${ }^{3}$ One-third of patients have a family history. Environmental factors include poor hygiene, variation in routine depilatory technique and use of antiperspirant. It starts as solitary or multiple isolated abscess without scarring or fistula formation. In the later stage, it presents with recurrent abscesses with sinus formation. In the final stage, it leads to broad involvement across a regional area with multiple interconnected sinus tracts and abscesses including subcutaneous tracts of infection. The patients in this stage have severe pain and unable to function properly. 
Medical treatment with antibiotics, analgesics and local antiseptic creams will only give short-term relief in these patients. They will have recurrent infections leading on to scarring and restriction of shoulder movements.

Surgical treatment options include incision and drainage of an abscess, excision of the involved skin and primary closure (in case of small area), wide excision with skin grafting, healing by secondary intension and use of local or regional flaps.

We describe a simple option- keystone design, islanded, perforator flap from the adjacent area for the cover of axillary defect after wide local excision of scarred, infected skin affected by hidradenitis suppurativa of axilla. We have treated 8 patients with this flap with good success.

\section{MATERIALS AND METHODS}

This case series involves study of 8 patients who underwent keystone flap for axillary defect following wide excision of skin with hidradenitis suppurativa from 2013 to 2015. Patient demographic data, medical histories, comorbidities, defect characteristics, hospitalisation, complications and follow-up were evaluated and presented as uncontrolled case series.

\section{Flap Planning and Design}

Wide local excision of the involved axillary skin along with $1 \mathrm{~cm}$ margin of normal skin was excised in all the patients. The length and width of the defect was measured intraoperatively. The maximum width was noted. The side of the defect with greater tissue laxity is chosen as the flap donor area. The incision made at 90 degrees at either end of the ellipse meets the curvilinear line of the flap's outer margin. One side of the elliptical defect serves as the inner arc of the keystone. The width of the flap was taken as same as the width of the defect. The flap was designed long enough to get bilateral V-Y advancement from both sides.

\section{Surgical Technique}

Incision is made all along the flap's boundary as marked before. The incision is deepened until deep fascia, which is also divided all along the outer border. Undermining of the flap border should not be done to prevent injury to fasciocutaneous and musculocutaneous perforators. Minimum undermining can be done on the inner side of the defect if there is any tension in the suture line. First suture was taken at the centre of the flap where there will be maximum tension. The remaining part of the flap is properly sutured to the defect, closure of the ' $\mathrm{Y}$ ' limb and rest of the flap on the outer border is done using standard technique. Keystone flap should be designed on that side of the defect having maximum skin expansibility. Double flaps may be required to cover larger defects or where there is less expansibility of adjacent skin. This flap survives based on perforators emerging from the underlying tissue. Suction drain should be used to facilitate proper drainage of collection.

\section{RESULTS}

Eight patients were included in the study. Ages of the subjects were ranging from 18 to 55 years with an average of 34.13 years. Six patients had bilateral involvement and remaining two patients had unilateral involvement of the disease. All of them had received medical treatment for more than 6 months without good result. Two patients in our study had known risk factor that was diabetes. Fourteen keystone flaps were done for these patients including unilateral and bilateral disease. Surgery was done in two stages for patients with bilateral involvement with a gap of 2 months. The average intraoperative time is 58.5 mins (range 35 - 75 mins). Suction drain was kept in all the cases for a period of 5 days. A padded dressing with aeroplane slab was applied in all the patients for 5 days to facilitate wound immobilisation and rest. Wound was inspected on $3^{\text {rd }}$ postoperative day. Antibiotics were given for 5 days. All the keystone flaps survived giving a durable cover to the axilla. However, two patients had wound infection, which was subsided with dressings and appropriate antibiotics. Mobilisation of the shoulder and elbow was initiated on $6^{\text {th }}$ day and the slab was discontinued. The patients were discharged on $7^{\text {th }}$ day with dressing and physiotherapy advice. The average duration of hospital stay of patients was 6.5 days. The sutures were removed after 2 weeks and patients were followed monthly for 6 months. All the flaps survived well with full range of shoulder movement and good symptomatic relief from pain and pus discharge. There was no recurrence in any of the cases over a period of 6 months.

\section{DISCUSSION}

Conservative management cures only a small percentage of patients. Those patients who are getting recurrent infections will require definitive surgical treatment. The surgical options available are excision and primary closure, excision and allowing the wound to heal by secondary healing, excision and skin grafting and wide excision followed by local or regional flap cover.

All these methods have their own merits and demerits. Excision and primary suturing will lead to insufficient excision leading to high chance of relapse of the disease. Primary suturing as well as healing by secondary intensions will result in restriction of shoulder mobility due to lack of soft tissue. Wide local excision and skin grafting will favour complete excision of the lesion, but may result in shoulder rigidity due to secondary contracture of the superficial thickness skin graft. Although split-skin grafting is a functionally an acceptable option, it does not give an ideal aesthetic outcome. ${ }^{1}$ Oliveira et al have suggested thoracodorsal fasciocutaneous flap cover the axillary defect. ${ }^{1}$ This fasciocutaneous flap consists of skin, subcutaneous fat and deep fascia, and the basis of this flap is blood supply from thoracodorsal artery. ${ }^{1}$ This is a fairly good technique; however, primary closure may be difficult after elevation of large flaps to cover extensive defects.

Geh and Niranjan promote the advanced double-opposing $\mathrm{V}-\mathrm{Y}$ fasciocutaneous flap as an alternative for axillary reconstruction. ${ }^{2}$ This method involves mobilisation of soft tissue on either side of the defect. Doppler localisation of the perforator and meticulous dissection and skeletalisation of the pedicle are essential.

Wan-Lin Teo et al explained about the use of lateral thoracic flap to cover axillary defect after excision of 
hidradenitis suppurativa. 4 They have done a two-staged procedure involving debridement, flap delay and vacuum aided dressing in the first stage and flap cover in the second stage. This flap provides a reliable flap to the defect, but the duration of hospitalisation is about 2 weeks.

The goal of axillary release is to achieve maximal shoulder movement, especially abduction. ${ }^{5}$ Aesthetic consideration is important as well to achieve good contour and texture of the axilla. 5 The keystone flap cover for axillary defect performed in our study achieves all of the above and the procedure is simple and easy to learn.

$\mathrm{AH}$ Schwabegger et al promoted the lateral thoracic fasciocutaneous island flap for axillary defects, which has many advantages- rapid dissection, avoidance of dog ears, mobility and range of motion and direct closure of the donor in a hidden area. ${ }^{6}$

Parascapular and scapular flaps were effectively used for reconstruction of defects in their arc of rotation, i.e. axilla, anterolateral chest and shoulder, whenever supple skin is needed for covering the defects and with functional improvement namely in range of motion of shoulder joint. ${ }^{7}$

Axillary defects after excision of hidradenitis suppurativa can also be covered by pedicled latissimus dorsi myocutaneous flaps. This flap can be used for large defects; however, this flap may be bulky and may require debulking procedure.

Elliot et al proposed posterior arm flap for reconstruction of axilla after hidradenitis excision. ${ }^{8}$ Cosmetic result of this flap is generally good. The flap having sufficient bulk to fill the hollow in the axilla on the left by high dissection, in contrast to the less than ideal after skin graft reconstruction. ${ }^{8}$

Varkarakis et al explains about a series of patients undergoing wide resection of axillary hidradenitis with reconstruction by Limber transposition flaps. ${ }^{9}$ Depending on the configuration of the defect, Limber transposition flaps were elevated from the chest wall on the posterior axillary margin. ${ }^{9}$

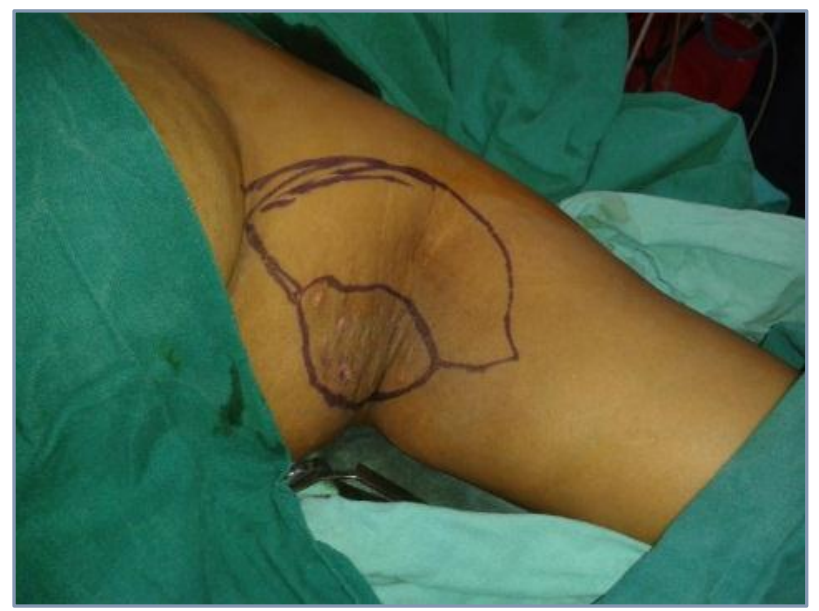

Figure 1. Intraoperative Skin Markings of Defect and Keystone Flap

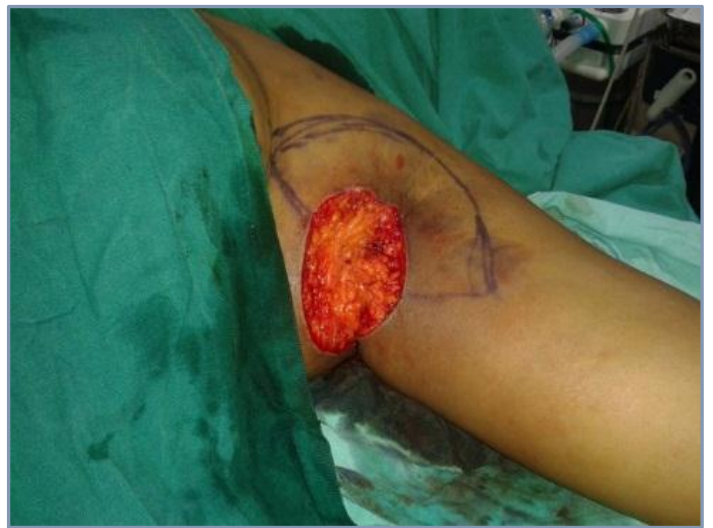

Figure 2. Local Excision of the Affected Skin

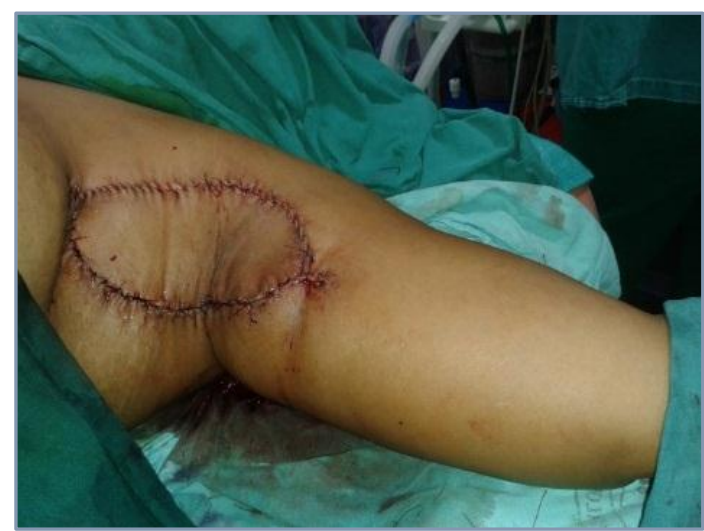

Figure 3. Immediate Post-operative Period after Excision of Defect and Suturing of the Flap

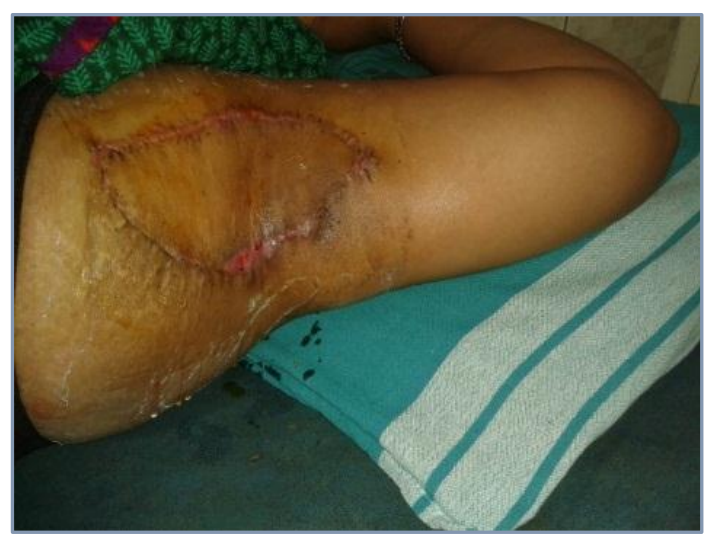

Figure 4. Post-operative Period (2 weeks) after Suture Removal with Primary Healing of Surgical Site

Keystone Flap is a Durable Flap. The advantages of Keystone Flap for the Reconstruction of Axilla are-

1. They are basically double V-Y advancement flaps and generate tissue from the adjacent lax area. Flap provides a durable cover to the axilla.

2. Keystone flaps can be elevated for any size of the defect. They bring lax tissue to the axilla favouring a normal range of movement to the shoulder.

3. There is no need for Doppler detection of perforators; flap base will invariably have good number of perforators.

4. The flap elevation technique is relatively simple, perforator location or skeletalisation is not needed and hence can easily be duplicated by young surgeons. 


\section{Jemds.com}

5. Compared to skin grafting, long-term immobilisation is not required and early physiotherapy can be started.

\section{CONCLUSION}

Keystone flap is a relatively simple local flap, which provides suitable cover to the axilla after excision of hidradenitis suppurativa and facilitates normal range of shoulder movement after surgery.

\section{REFERENCES}

[1] Eleuterio JC, De Oliveira T, De Souza A, et al. Thoracodorsal fasciocutaneous flap in the treatment of hidradenitis suppurativa: a case report and literature review. Rev Bras Cir Plast 2012;27(1):170-3.

[2] Geh JLC, Niranjan NS. Perforator-based fasciocutaneous flaps for the reconstruction of axillary defects following excision of hidradenitis suppurativa. British Journal Plastic Surgery 2002;55(2):124-8.

[3] Desai N, Van Der Zee HH, Jemec BEG. Hidradenitis suppurativa. Rooks textbook of Dermatology. 9th edn. Wiley-Blackwell 2016;3:92.1-11.

[4] Teo WL, Obg YS, Tan BK. Radiacal surgical excision and use of Lateral thoracic flap for intractable axillary hidradenitis suppurativa. Archives of Plastic Surgery 2012;39(6):663-6.

\section{Original Research Article}

[5] Devi SR, Baishya J. Management of post burn axillary contracture along with breast contracture: our experience. Indian Journal of Burns 2012;20(1):23-9.

[6] Schwabegger AH, Herczeg E, Piza H. The lateral thoracic fasciocutaneous island flap for treatment for treatment of recurrent hidradenitis axillaris suppurativa and other axillary skin defects. British Journal of Plastic Surgery 2000;53(8):676-8.

[7] Abood MH, Abdilkarim DA. Parascapular pedicle fasciocutaneous flaps for regional reconstruction, evaluation study. Journal of US-China Medical Sciences 2011;8(10),(Serial No.83):626-37 D.

[8] Elliot D, Kangesu L, Bainbridge C, et al. Reconstruction of axilla with a posterior arm fasciocutaneous flap. British Journal of Plastic Surgery 1992;45(2):101-4.

[9] Varkarakis G, Daniels J, Coker C, et al. Treatment of axillary hidradenitis with transposition flaps: a 6-year experience. Annals of Plastic Surgery 2010;64(5): 592-4. 